\title{
Analysis of TSC2 stop codon variants found in tuberous sclerosis patients
}

\author{
Miriam A Goedbloed ${ }^{1}$, Mark Nellist ${ }^{1}$, Brenda Verhaaf ${ }^{1}$, Arnold JJ Reuser ${ }^{1}$, Dick Lindhout ${ }^{2}$, \\ Lone Sunde $^{3}$, Senno Verhoef ${ }^{4,5}$, Dicky JJ Halley ${ }^{1}$ and Ans MW van den Ouweland ${ }^{*, 1}$ \\ ${ }^{1}$ Department of Clinical Genetics, Erasmus University and Dijkzigt Academic Hospital, Dr. Molewaterplein 50, \\ 3015 GE Rotterdam, The Netherlands; ${ }^{2}$ Department of Medical Genetics, University Medical Centre Utrecht, \\ 3508 AB Utrecht, The Netherlands; ${ }^{3}$ Aarhus Kommunehospital, DK-800 Aarhus C, Denmark; ${ }^{4}$ Department of \\ Clinical Genetics, Academic Medical Centre/Family Cancer Clinic, Meibergdreef 15, 1105 AZ Amsterdam; \\ ${ }^{5}$ The Netherlands Cancer Institute, Plesmanlaan 121, 1066 CX Amsterdam, The Netherlands
}

Tuberous sclerosis complex (TSC) is an autosomal dominant disorder caused by mutations to the TSC1 and TSC2 tumour suppressor genes. We detected two sequence changes involving the TSC2 stop codon and investigated the effects of these changes on the expression of tuberin, the TSC2 gene product, and on the binding between tuberin and the TSC1 gene product, hamartin. While elongation of the tuberin open reading frame by 17 amino acids did not interfere with tuberin-hamartin binding, a longer extension prevented this interaction. Our data illustrate how functional protein assays can assist in the verification and characterisation of disease-causing mutations. European Journal of Human Genetics (2001) 9, 823-828.

Keywords: tuberous sclerosis; tuberin; hamartin; stop codon mutations

\section{Introduction}

Tuberous sclerosis complex (TSC) is characterised by the development of a variety of lesions in many different tissues. The most important symptoms in TSC patients include seizures, mental handicap, behavioural problems, renal dysfunction and dermatological abnormalities. The neurological problems are most likely caused by the presence of cortical tubers, subependymal nodules and giant cell astrocytomas in the brain. ${ }^{1}$

TSC is a genetic disease that is inherited as an autosomal dominant trait, and is caused by mutations in either the TSC1 or TSC2 tumour suppressor genes. The TSC1 and TSC2 genes encode two large, unrelated proteins called hamartin and tuberin respectively. ${ }^{2,3}$ Hamartin and tuberin interact, and it has been suggested that formation of a tuberin-hamartin

${ }^{*}$ Correspondence: Dr. A.M.W. van den Ouweland, Department of Clinical Genetics, Erasmus University, 3015 GE Rotterdam, The Netherlands. Tel: 3110 4087197; Fax: 3110 4089489;

E-mail: vandenouweland@kgen.fgg.eur.nl.

Received 30 May 2001; revised 30 August 2001; accepted 5 September 2001 complex may be important for the correct functioning of both proteins. ${ }^{4-6}$ We detected two sequence changes involving the TSC2 stop codon and investigated the effects of these changes on the expression of tuberin and on the binding between tuberin and hamartin. Our results illustrate how investigations into protein function can assist in the verification and characterisation of disease-causing mutations.

\section{Materials and methods}

Mutation screening

Individual TSC1 and TSC2 exons were amplified by PCR, and combined single strand conformation polymorphism (SSCP)/ heteroduplex analysis was performed using the Pharmacia GenePhor electrophoresis system. Variant patterns were investigated by direct sequencing of the PCR products. ${ }^{7}$

\section{Site-directed mutagenesis}

The construction of yeast two-hybrid and mammalian expression vectors containing TSC1 and TSC2 cDNAs has been described previously. ${ }^{4,5}$ To introduce the TSC2 5440delTG and 5438delTGTG changes, site-directed muta- 
genesis was performed using the Stratagene QuickChange kit. Primers 5'-cttcaccgagtttgtgaggccggggccctc- 3 ' and 5'gagggcccogectcacaaactcggtgaag- $3^{\prime}$ were used to introduce the TSC2 5440delTG change; 5'-cttcaccgagttgaggccggggccetc-3' and 5'-gagggcccoggctcaaactcggtgaag-3' for the TSC2 5438delTGTG variant. All the constructs generated for this study were sequenced completely to ensure no additional changes had been introduced during the mutagenesis procedure.

\section{Transfection experiments}

Expression constructs were transfected into COS-7 cells with lipofectAMINE and PLUS reagent, as recommended by the manufacturer (Life Technologies). Forty-two hours after transfection, cells were analysed by immunofluorescence microscopy, as described previously, ${ }^{4,5}$ or harvested for coimmunoprecipitation and immunoblotting experiments. Cells from one $30 \mathrm{~cm}^{2}$ plate were washed with phosphatebuffered saline, collected by scraping and pelleted by centrifugation at $2500 \mathrm{rpm}$ for $5 \mathrm{~min}$ at $4^{\circ} \mathrm{C}$. The cells were lysed in $700 \mu \mathrm{l}$ TNE buffer (40 mM Tris- $\mathrm{HCl} \mathrm{pH} 8.0,150 \mathrm{~mm}$ $\mathrm{NaCl}, 1 \mathrm{~mm}$ EDTA, 1\% Triton X-100) containing protease inhibitors (Complete; Roche Molecular Biochemicals), and the nuclei and cellular debris were removed by centrifugation at $10000 \mathrm{~g}$ for $10 \mathrm{~min}$ at $4{ }^{\circ} \mathrm{C}$. The supernatant was recovered and used for Western blotting and coimmunoprecipitation experiments. For the coimmunoprecipitation experiments, the cleared COS cell lysates were incubated with $2 \mu \mathrm{l}$ hamartin-specific antiserum for $90 \mathrm{~min}$ on ice before the addition of $30 \mu \mathrm{l}$ of a $50 \%$ suspension of Protein A-Sepharose beads. After gentle rotation at $4^{\circ} \mathrm{C}$ for a further $90 \mathrm{~min}$, the beads were washed extensively with TNE buffer and finally resuspended in $75 \mu \mathrm{l}$ SDS-PAGE loading buffer. Immunoblotting was performed according to standard procedures. ${ }^{8}$

\section{Yeast two-hybrid experiments}

Yeast host strain Y190 was cotransformed with $0.3-0.5 \mu \mathrm{g}$ of both the GAL4 activation and binding domain constructs, according to the protocols recommended by the supplier (Clontech). Transformants were plated on media lacking tryptophan and leucine, and on media lacking histidine, tryptophan and leucine (-hlt). $\beta$-Galactosidase filter assays were performed on colonies growing on -hlt media.

\section{Detection of TSC2 transcripts}

Total RNA was prepared from cultured patient fibroblasts using the RNAEasy protocol (Qiagen). Reverse transcription was performed on $1 \mu \mathrm{g}$ total RNA using random hexanucleotide primers, according to standard protocols. ${ }^{9}$ To distinguish the RT-PCR cDNA products from possible contaminating genomic sequences, PCR was performed with primers specific for TSC2 exons 40 (5'-tcaagcggctccgccagcgg-3') and 41 (5'gtctgtgcactggggtcaggac-3'). The 269 base-pair wild-type and 267 base-pair mutant cDNA PCR products were distinguished by allele specific oligonucleotide hybridisation with oligo- nucleotides 5'-tttgtgtgaggccg-3' and 5'-gtttgtgaggccgg-3', specific for the TSC2 wild-type and TSC2 5440delTG sequences respectively.

\section{Results}

We screened $>470$ TSC patients for TSC1 and TSC2 mutations by combined SSCP/heteroduplex analysis. In one Dutch TSC patient, a deletion of two nucleotides at the stop codon of the TSC2 gene (TSC2 5440delTG) was identified, consistent with the finding of chromosome 16p13 loss of heterozygosity in a gingival fibroma from this patient (unpublished results). No additional TSC1 or TSC2 changes were detected in the patient and the TSC2 5440delTG deletion was not detected in either unaffected parent, indicating that the TSC2 5440delTG deletion was de novo and most likely the causative mutation. The TSC2 5440delTG deletion disrupts the normal TSC2 stop codon resulting in elongation of the open reading frame.

The segregation of TSC through a two generation Danish family is shown in Figure 1. The index patient, individual 2057, has facial angiofibroma, ungual fibroma, hypomelanotic macules, subependymal nodules as revealed by cranial tomography (CT), cortical tubers as revealed by magnetic resonance imaging, suffers from epilepsy and is mildly mentally retarded. Individual 2055 has facial angiofibroma, ungual fibroma and hypomelanotic macules; individual 2044 has facial angiofibroma, ungual fibroma, hypomelanotic macules, and subependymal nodules as revealed by CT. All the other family members were classified as unaffected upon clinical examination except individual 1494 (cross-hatched diamond) who was too young for a definitive diagnosis to be made, although no signs of TSC were detected. Combined SSCP/heteroduplex analysis of the index patient resulted in the detection of two sequence alterations: 814delACT in the TSC1 gene, and 5438delTGTG in the TSC2 gene. The TSC1 814 delACT change was described previously ${ }^{7}$ and is predicted to lead to the replacement of the amino acids asparagine and phenylalanine at positions 198 and 199 of hamartin, the TSC1 gene product, with a single isoleucine. The TSC2 5438delTGTG deletion disrupts the normal TSC2 stop codon and extends the TSC 2 open reading frame by 17 codons, before a new stop codon is reached at nucleotide 5491 .

We compared the effect of the TSC2 5440delTG and TSC2 5438delTGTG changes on the expression and function of tuberin, the TSC2 gene product. In transfected cells, the TSC2 5438delTGTG encoded tuberin variant was localised to the cytoplasm by immunofluorescence microscopy, indistinguishable from wild-type tuberin (data not shown). As shown in Figure 2, the TSC2 5438delTGTG-derived tuberin variant and hamartin could be coimmunoprecipitated from COS cells cotransfected with full-length TSC1 and TSC2 5438delTGTG expression constructs.

Immunofluorescence microscopy indicated that the transfection efficiency of the TSC2 5440delTG expression 


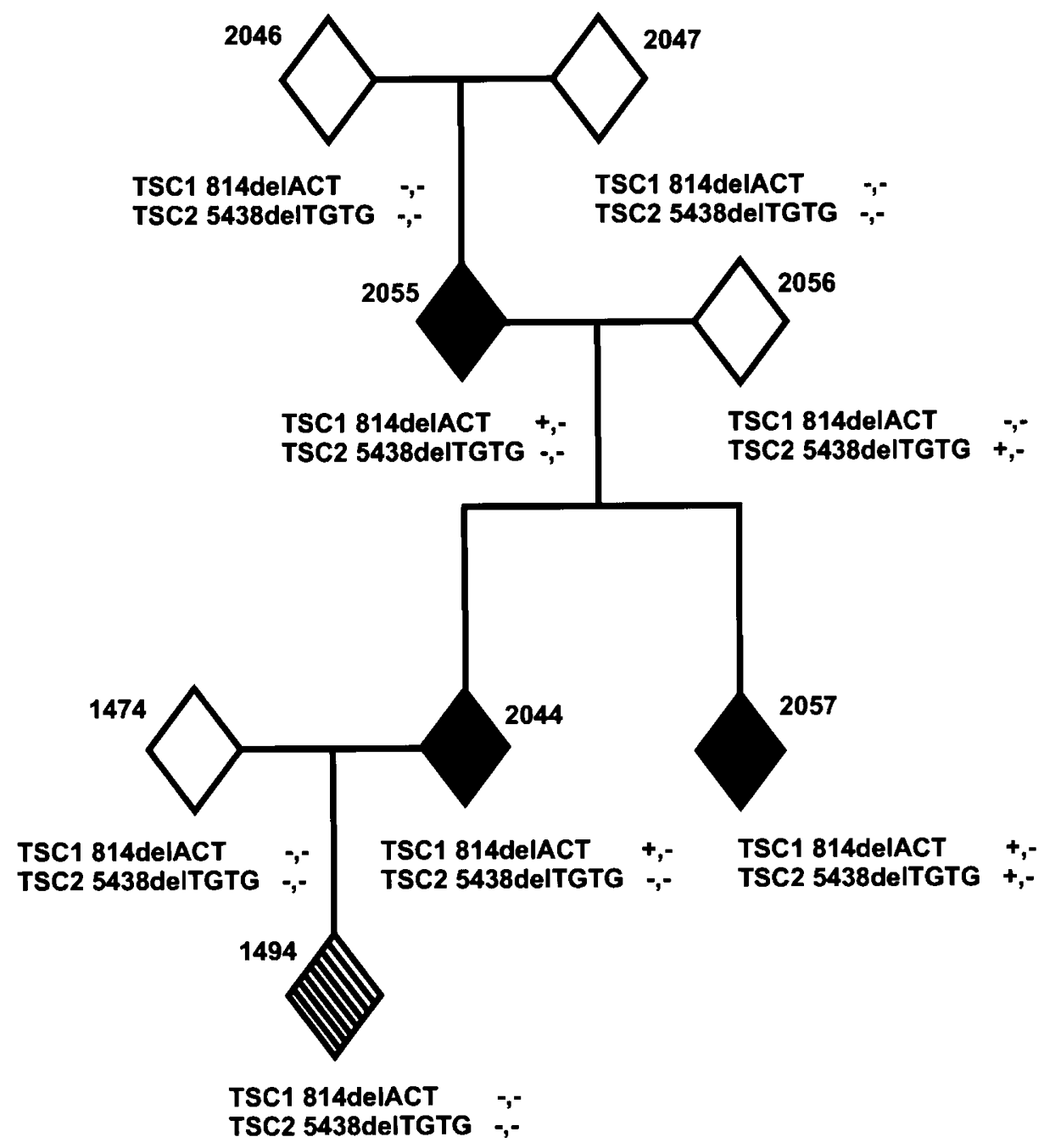

Figure 1 Segregation of TSC1 814delACT and TSC2 5438delTGTG through a family with TSC. The four generation Danish family segregating TSC as well as variants in both the TSC1 and TSC2 genes is shown. In each case, $a^{\prime}+{ }^{\prime}$ indicates the presence of the variant allele (TSC1 814delACT or TSC2 5438delTGTG) while '- ' represents the normal, wild-type allele. The solid diamonds represent affected individuals 2055, 2044 and 2057. Individual 2055 has facial angiofibroma, ungual fibroma and hypomelanotic macules; individual 2044 has facial angiofibroma, ungual fibroma, hypomelanotic macules and an abnormal CT; individual 2057 has facial angiofibroma, ungual fibroma, hypomelanotic macules, an abnormal CT, suffers from epilepsy and is mildly mentally retarded. All the other family members were classified as unaffected upon clinical exam except individual 1494 (cross-hatched diamond) who is still too young for a definitive diagnosis to be made.

construct was comparable with both the wild-type and TSC2 5438delTGTG expression constructs (approximately 50\% cells expressing exogenous tuberin), and that the cytoplasmic localisation of the protein was also unaffected. However, as shown in Figure 2, the TSC2 5440delTG protein product migrated more slowly through SDS-PAGE gels than the wild-type tuberin protein. In addition, the level of expression was lower and we were not able to detect the protein in the hamartin coimmunoprecipitate, suggesting that the TSC2 5440delTG encoded tuberin variant was either unable to bind hamartin or too unstable to form a detectable complex.

We investigated whether the TSC2 5440delTG mutation also prevented the tuberin-hamartin interaction in the yeast two-hybrid system. The results of the yeast two-hybrid experiments are shown in Figure 3. No large yeast colonies grew on -hlt plates and no $\beta$-galactosidase activity could be detected in yeast cotransformed with a GAL4 binding domain-TSC2 5440delTG full-length cDNA construct and the GAL4 activation domain-TSC1 construct. In contrast, $\beta$ - 


\section{a. Lysate \\ b. Hamartin IP

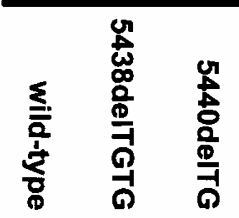

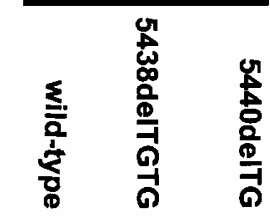 \\ Tuberin

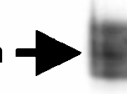 (1)
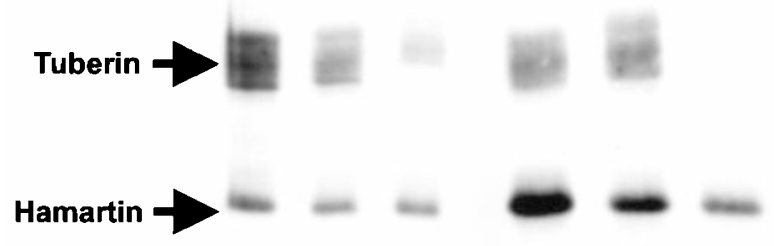

Figure 2 Effect of TSC2 stop codon mutations on coimmunoprecipitation of the tuberin-hamartin complex. Tuberin-hamartin complexes were immunoprecipitated, with antibodies specific for hamartin, from COS cells cotransfected with TSC1 and either wild-type TSC2, TSC2 5438delTGTG or TSC2 5440delTG. Expression of hamartin and tuberin, and the presence of the tuberin-hamartin complex was investigated by Western blotting. (a) Western blot analysis of the COS cell lysates. Hamartin, wild-type tuberin and both the 5438delTGTG and 5440delTG tuberin variants were expressed. (b) Analysis of the hamartin immunoprecipitates. Both wild-type tuberin and the 5438delTGTG variant are coimmunoprecipitated. In contrast, the 5440delTGTG variant does not form a coimmunoprecipitable complex with hamartin.

galactosidase activity was detected in yeast cotransformed with a GAL4 activation domain-TSC1 construct and GAL4 binding domain constructs encoding either wild-type fulllength TSC2 or the TSC2 5438delTGTG variant. Consistent with the coimmunoprecipitation experiments, the tuberinhamartin interaction was not affected by the TSC2 5438delTGTG change, but was prevented by the TSC2 5440delTG mutation.

To study the effect of the TSC2 5440delTG mutation on the expression of both the TSC2 mRNA and tuberin in vivo, fibroblasts from the index patient were analysed. First we investigated whether the TSC2 5440delTG mRNA was transcribed. RT-PCR of the TSC2 mRNA was followed by ASO hybridisation to distinguish between the normal and mutant transcripts. Both the wild-type and 5440delTG transcripts were detected in RNA prepared from fibroblasts from the patient, indicating that the TSC2 5440delTG mutant mRNA was expressed (data not shown). No TSC2 5440delTG mRNA was detected in a control RNA sample. By Western blot analysis there was no apparent difference between tuberin expression in fibroblasts from the 5440delTG patient and fibroblasts from an individual without TSC. We could not distinguish the TSC2 5440delTG encoded variant, if present, from wild-type tuberin (data not shown).

\section{Discussion}

In a diagnostic setting it can be difficult to distinguish between disease-causing mutations and rare, non-pathogenic polymorphisms. For TSC this is particularly true for changes that do not lead to premature truncation of the TSC1 or TSC2 gene products. The TSC2 5440delTG and TSC2 5438delTGTG deletions identified in this study both cause elongation of the TSC2 open reading frame and are good examples of changes that at first glance are difficult to classify as being responsible for TSC. Both deletions are interesting in that they both destroy the normal TSC2 stop codon. Similar changes have not been described previously in TSC patients, and stop codon mutations have not been encountered frequently in other inherited conditions, eg hereditary breast/ovarian cancer, either (http://www.nhgri.nih.gov/Intramural_research/Lab_transfer/Bic/). Exon 41 of the TSC2 gene is also notable for a relative paucity of TSC-associated mutations. One of the reasons for this may be that deletion of only a few amino acids at the carboxy terminal of such a large protein as tuberin may not significantly affect its function.

The TSC2 3' untranslated region (UTR) is relatively short ( $\sim 100$ nucleotides), and the TSC2 5440delTG mutation deletes the normal TSC2 stop codon extending the open reading frame through the entire 3 ' UTR, into the polyadenine tail. Between the normal TSC 2 stop codon and the polyadenylation site, where transcription terminates prior to polyadenylation, no new in-frame stop codon is encountered. It was not immediately clear what effect deletion of the stop codon would have on tuberin translation. The most obvious assumption was that any TSC 2 mRNA lacking a stop codon would not be efficiently translated or released from the ribosome, leading to a reduction in tuberin expression. However, multiple polyadenylation sites are utilised during transcription of the TSC 2 mRNA $^{3}$ and we could not exclude the possibility that differential processing of the TSC2 5440delTG mRNA would result in tuberin biosynthesis. The TSC2 5440delTG mutation extends the tuberin open reading frame and it was therefore possible that active tuberin could still be produced from the TSC2 5440delTG mRNA and that the deletion was not responsible for TSC in this patient. This possibility was highlighted when we identified a very similar deletion, TSC2 5438delTGTG, in another TSC patient that also resulted in extension of the tuberin open reading frame but which segregation analysis suggested was not pathogenic. The similarities between the TSC2 5438delTGTG deletion and the TSC2 5440delTG deletion raised concerns over whether we could definitively classify one as a mutation and the other as a rare polymorphism. Therefore, we sought additional evidence for the pathogenicity or otherwise of both changes by investigating the effect of elongation of the tuberin open reading frame on tuberin expression and on the tuberin-hamartin interaction.

Western blot analysis of transfected cells expressing the TSC2 5440delTG-encoded tuberin variant indicated that the mutant protein was only detectable at reduced levels and 


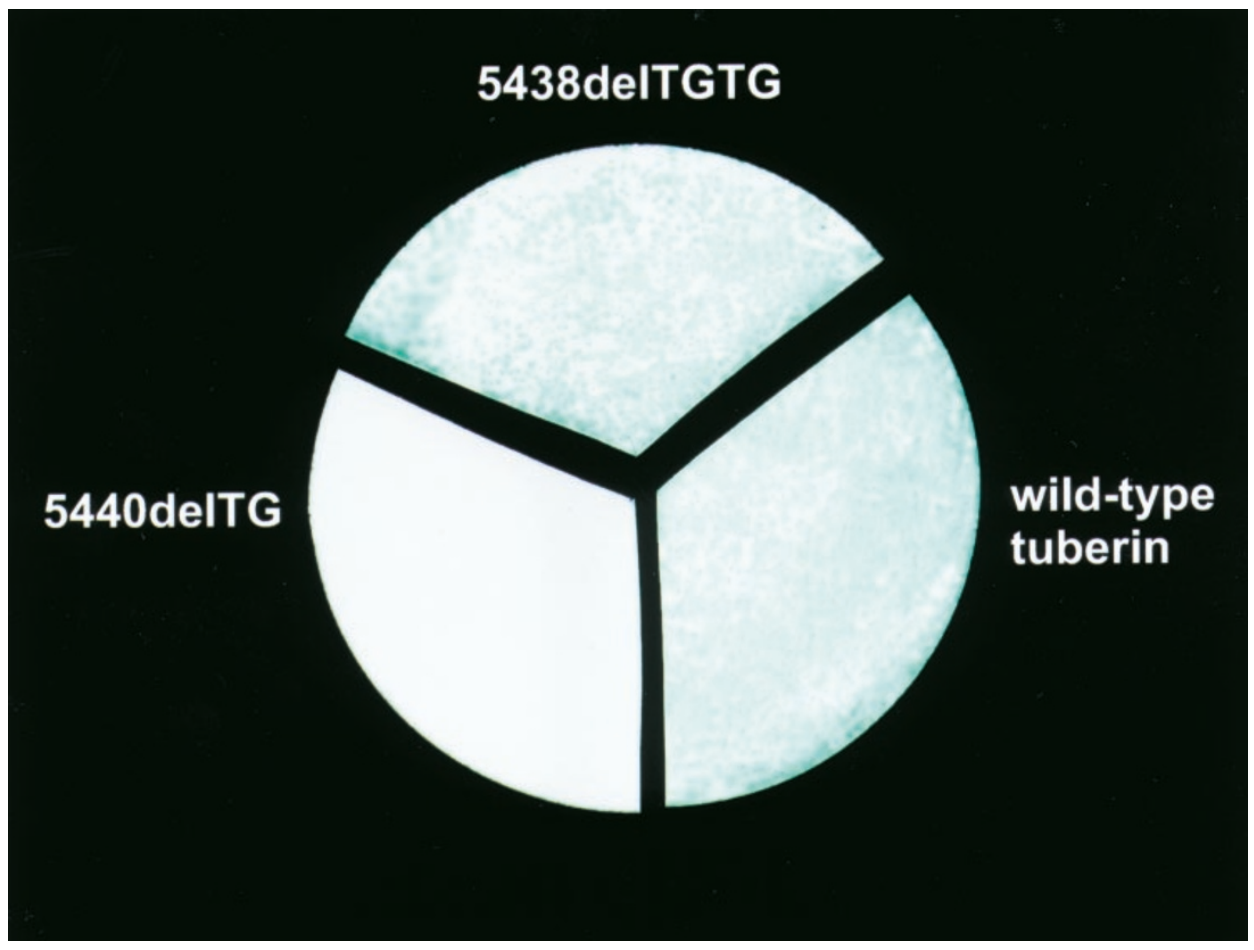

Figure 3 Yeast two-hybrid analysis of the effect on the interaction between tuberin and hamartin of TSC2 5440 delTG and 5438delTGTG. GAL4 binding domain constructs encoding the TSC2 wild-type, 5440delTG and 5438delTGTG full-length cDNAs were cotransformed into yeast Y190 cells with a GAL4 activation domain-TSC1 fusion construct. Cotransformants were plated on -hlt medium and tuberin-hamartin binding confirmed by a $\beta$-galactosidase colony lift assay. Both the TSC2 wild-type and the 5438 delTGTG variant interact with hamartin (blue colonies; grew well on -hlt medium) while, in contrast, the 5440delTG variant does not interact with hamartin (white colonies; very limited growth on -hlt medium).

migrated more slowly through SDS-PAGE gels than wildtype tuberin. The reduced mobility of the mutant protein was consistent with elongation of the open reading frame while the reduced amount of protein detected on the Western blot suggested that the mutant protein was less efficiently translated and/or less stable than wild-type tuberin. The expression constructs used for the transfection and yeast twohybrid experiments contained a relatively short $(\sim 30$ nucleotide) poly A tail, followed by vector sequences. The mRNA transcripts produced from these constructs, therefore, also contain stop codons derived from the vector sequences. The slower migration of the TSC2 5440delTG encoded protein is consistent with this size increase.

The RT-PCR/ASO analysis indicated that the TSC2 5440delTG mRNA was transcribed. However, we could not distinguish a more slowly migrating tuberin variant in fibroblasts from the patient with the TSC2 5440delTG mutation. This may be due to either reduced expression of the mutant tuberin, instability, or both. In vivo, a poly A tail 100-200 nucleotides long is attatched to most mRNAs $^{10}$ and this may interfere with synthesis and/or release of the nascent protein chain from the ribosome when no stop codon is present, preventing expression of tuberin from the TSC2 5440delTG mRNA.
The transfection and yeast two-hybrid experiments demonstrated that even if protein is produced from the TSC2 5440delTG allele it is unlikely to be active since we could not detect any interaction with hamartin. It is possible that the mutant protein is too unstable to form detectable levels of the tuberin-hamartin complex. Alternatively, the additional carboxy-terminal amino acids may alter the conformation of tuberin, interfering with hamartin binding. Although neither the coimmunoprecipitation nor yeast two-hybrid experiments indicated whether the affect of the TSC2 5440delTG mutation was only to reduce tuberin expression or whether the additional carboxy terminal domain interfered specifically with tuberin-hamartin binding, both assays demonstrated a clear effect, distinct from both wild-type tuberin and the TSC2 5438delTGTG variant.

In contrast to the TSC2 5440 delTG mutation, both tuberin expression and binding were unaffected by the additional 17 amino acids present due to the TSC2 5438delTGTG change. The TSC2 5438delTGTG change was identified in a Danish family with TSC. In the index patient, individual 2057, two changes were found: TSC1 814delACT and TSC2 5438delTGTG. It was not possible to determine whether the TSC1 814delACT in-frame deletion was the causative mutation, or whether the TSC2 5438delTGTG frameshift was 
responsible for TSC. Other TSC families likely to be segregating more than one mutation have been described, ${ }^{11}$ as well as a sporadic TSC case where multiple de novo sequence variants have been identified. ${ }^{12} \mathrm{~A}$ more detailed analysis of the segregation of the TSC1 814delACT and TSC2 5438delTGTG changes through the family was undertaken. Only the TSC1 814 delACT variant segregated with TSC. The TSC2 5438delTGTG change was inherited by the index patient from the unaffected parent (individual 2056). Neither an affected sibling (individual 2044) nor the affected parent (individual 2055) of the index patient carried the TSC2 5438delTGTG change, making it unlikely that this change was responsible for TSC in the index patient. Individual 2056, who also carried the TSC2 5438delTGTG change, has no clinical signs of TSC and no family history of the disease. We did not detect the TSC2 5438delTGTG change in any of the other $>470$ TSC patients screened, indicating that it is most likely a rare nonpathogenic TSC2 sequence variant. However, we have not tested a large number of Danish samples and it is possible that the polymorphism is restricted to this particular population.

Analysis of the TSC2 5438delTGTG variant provided additional evidence that the TSC2 5440delTG mutation was responsible for the reduction in tuberin expression and the inhibition of tuberin-hamartin binding and that the observed effects were unlikely to be artefacts of the assay systems used.

It is possible that the proposed tuberin rap1 and rab5 GAP activities could be affected by the 5438 delTGTG change since both the predicted rap1GAP-related and rabaptin5-binding domains are located close to the carboxy terminus. ${ }^{13,14}$ The affected individual 2057 who carries both the TSC1 814delACT mutation and TSC2 5438delTGTG variant has a more severe phenotype than both the affected parent (individual 2055) and the affected sibling (individual 2044) (Figure 1). However, whether this is due to the TSC2 5438delTGTG or is a consequence of other factors that contribute to the wide phenotypic variation recognised in TSC is not clear. The phenotypes of individuals 2055 and 2044 are consistent with recent data suggesting that TSC1 mutations are more often associated with a milder form of TSC. ${ }^{15}$

In summary, during screening for TSC1 and TSC2 mutations in a large cohort of TSC patients we identified two patients with small deletions involving the normal TSC2 stop codon. We studied the effects of both sequence changes on tuberin, the TSC2 gene product. The TSC2 5438delTGTG polymorphism had no discernible effect on either tuberin expression or hamartin-tuberin binding. In contrast, the TSC2 5440delTG mutation reduced tuberin expression levels and inhibited formation of the tuberin-hamartin complex in vitro. Characterisation of the protein products of the two TSC2 stop codon variants supported the conclusions of the genetic investigations and suggests that similar assays may be of use in other cases where subtle TSC1 and TSC2 sequence changes have been detected and doubt remains as to whether the detected changes are pathogenic mutations. Although the approach described in this study is not feasible for routine analysis of TSC patient mutations, it does indicate how functional assays of tuberin and hamartin can be useful in a diagnostic setting.

\section{Acknowledgments}

The authors would like to thank the TSC families involved in this study, and their attending physicians, for their participation and cooperation, and $C$ Withagen-Hermans for performing the loss of heterozygosity study. Financial support was provided by the Tuberous Sclerosis Alliance (USA), Noortman b.v., Maastricht and the Nationaal Epilepsie Fonds (Netherlands).

\section{References}

1 Gomez MG, Sampson JR, Holets Whittemore V: Tuberous Sclerosis Complex. In: Developmental Perspectives in Psychiatry. 3rd edition. New York and Oxford, Oxford University Press, 1999.

2 van Slegtenhorst M, de Hoogt R, Hermans C et al. Identification of the Tuberous Sclerosis gene TSC1 on chromosome 9q34. Science 1997; 277: 805-808.

3 The European Chromosome 16 Tuberous Sclerosis Consortium: Identification and characterization of the tuberous sclerosis gene on chromosome 16. Cell 1993; 75: 1305-1315.

4 van Slegtenhorst M, Nellist M, Nagelkerken B et al. Interaction between hamartin and tuberin, the TSC1 and TSC2 gene products. Hum Mol Genet 1998; 7: $1053-1057$.

5 Nellist M, van Slegtenhorst MA, Goedbloed M, van den Ouweland AMW, Halley DJJ, van der Sluijs P: Characterization of the cytosolic tuberin-hamartin complex. J Biol Chem 1999; 274: $35647-35652$.

6 Plank TL, Yeung RS, Henske EP: Hamartin, the product of the tuberous sclerosis 1 (TSC1) gene, interacts with tuberin and appears to be localized to cytoplasmic vesicles. Cancer Res 1998; 58: $4766-4770$.

7 van Slegtenhorst M, Verhoef S, Tempelaars A et al: Mutation spectrum of the TSC1 gene in a cohort of 225 tuberous sclerosis complex patients; no evidence for genotype-phenotype correlation. J Med genet 1999; 36: 285 - 289.

8 Sambrook J, Fritsch EF, Maniatis T: Molecular Cloning: A Laboratory Manual. 2nd edition. Cold Spring Harbor, Cold Spring Harbor Laboratory Press, 1989.

9 Kawasaki ES: PCR Protocols: A Guide to Methods and Applications. Academic Press, London, 1990.

10 Alberts B, Bray D, Lewis J, Raff M, Roberts K, Watson JD: Molecular Biology of the Cell. 3rd edition. New York and London, Garland Publishing Inc., 1994.

11 Young JM, Burley MW, Jeremiah SJ, Jeganathan D, Ekong R, Osborne JP, Povey S: A mutation screen of the TSC1 gene reveals 26 protein truncating mutations and 1 splice site mutation in a panel of 79 tuberous sclerosis patients. Ann. Hum. Genet. 1998; 62: $203-213$.

12 Jones AC, Shyamsundar MM, Thomas MW et al: Comprehensive mutation analysis of TSC1 and TSC2 and phenotypic correlations in 150 families with tuberous sclerosis. Am. J. Hum. Genet. 1999; 64: $1305-1315$.

13 Wienecke R, Konig A, DeClue JE: Identification of tuberin, the tuberous sclerosis-2 product. J Biol. Chem. 1995; 270: 16409 16414.

14 Xiao GH, Shoarinejad F, Jin F, Golemis EA, Yeung RS: The tuberous sclerosis-2 gene product, tuberin, functions as a rab5 GTPase activating protein (GAP) in modulating endocytosis. J. Biol. Chem. 1997; 272: 6097-6100.

15 Dabora SL, Jozwiak S, Franz DN et al.: Mutational analysis in a cohort of 224 tuberous sclerosis patients indicates increased severity of TSC2, compared with TSC1, disease in multiple organs. Am J Hum Genet 2001; 68: 64-80. 